\title{
A Chattering Free Discrete-Time Global Sliding Mode Controller for Optoelectronic Tracking System
}

\author{
Yan Ren, ${ }^{1,2}$ Zhenghua Liu, ${ }^{1}$ Xiaodong Liu, ${ }^{3,4}$ and Yu Zhang ${ }^{5}$ \\ ${ }^{1}$ School of Automation Science and Electrical Engineering, Beihang University, Beijing 100191, China \\ ${ }^{2}$ Information Engineering School, Inner Mongolia University of Science and Technology, Baotou 014010, China \\ ${ }^{3}$ Beijing Aerospace Automatic Control Institute, Beijing 100854, China \\ ${ }^{4}$ National Key Laboratory of Science and Technology on Aerospace Intelligence Control, Beijing 100854, China \\ ${ }^{5}$ Beijing Institute of Space Launch Technology, Beijing 100076, China
}

Correspondence should be addressed to Xiaodong Liu; k.start@163.com

Received 25 July 2013; Accepted 3 October 2013

Academic Editor: Xudong Zhao

Copyright (c) 2013 Yan Ren et al. This is an open access article distributed under the Creative Commons Attribution License, which permits unrestricted use, distribution, and reproduction in any medium, provided the original work is properly cited.

\begin{abstract}
Aiming at the uncertainties including parameter variations and external disturbances in optoelectronic tracking system, a discretetime global sliding mode controller (DGSMC) is proposed. By the design of nonlinear switching function, the initial state of control system is set on the switching surface. An adaptive discrete-time reaching law is introduced to suppress the high-frequency chattering at control input, and a linear extrapolation method is employed to estimate the unknown uncertainties and commands. The global reachability for sliding mode and the chattering-free property are proven by means of mathematical derivation. Numerical simulation presents that the proposed DGSMC scheme not only ensures strong robustness against system uncertainties and small tracking error, but also suppresses the high-frequency chattering at control input effectively, compared with the SMC scheme using conventional discrete-time reaching law.
\end{abstract}

\section{Introduction}

Optoelectronic tracking system is a kind of servo system with high accuracy in position tracking. The performance of the system is usually badly affected by parameter perturbations and external disturbances including nonlinear friction load inertia torque and [1]. In the existence of the above system uncertainties, how to ensure beacon beam accurately aim at target has become a hot topic in oriented energy weapons, free space optical communication, and adaptive optics fields [2]. As a result, developing the control approaches to solve this problem is necessary for optoelectronic tracking system.

Sliding mode control (SMC) is a robust control method being suitable for linear system and nonlinear system [3]. From the theoretical perspective, because sliding mode can be designed according to our demands in advance, and the sliding mode motion is insensitive to parameter variations and external disturbances of control system, SMC has attracted more attentions and been applied in a wide range [4-7]. Therefore, SMC theory can be employed when we design a robust controller for the optoelectronic tracking system.

However, the control system possesses insensitivity to parameter variations and external disturbances merely on sliding mode. That is, if the reaching time to the sliding surface is shortened, the dynamic property and the robust property of control system will be effectively improved. Subsequently, how to shorten the reaching time is an important direction in SMC research. Reaching law approach $[8,9]$ can effectively improve the dynamic quality of reaching motion through artificial trajectory design, so the reaching time to sliding surface is shortened. Another idea, global sliding mode control (GSMC), which can make system trajectory initially set on the sliding surface by constructing nonlinear switching function and then the initial reaching motion is eliminated, has aroused researchers' interest [1013]. Therefore, the combined use of reaching law and nonlinear switching function is beneficial for the global robustness of control system and is introduced to construct controller for the optoelectronic tracking system in this paper. 
In conventional SMC methods, the gain of signum term is usually regarded as a constant. Moreover, when the gain of signum term is too large, the high-frequency chattering at control input is intense. In contrary, the reachability for sliding mode may not be satisfied. Therefore, seeking for adaptive algorithms to acquire the gain of signum term is crucial in SMC application. As a result, some researchers were inclined to use intelligent schemes to get the adaptive gain, such as [14-17]. However, the comprehensive property with respect to engineering practicability and good effectiveness has become an important factor in restricting their development. In addition, with the appearance and the development of MCU and DSP, the modularization and the digitalization of servo control system are easily achieved. Because the control algorithm is usually realized by digital computer, it is necessary to study SMC in discrete domain. Therefore, in order to guarantee the engineering practicability (with respect to simple and discrete-time designs) and the method effectiveness (with respect to small tracking error, strong robustness against unknown and varying disturbances, and smooth control input), we intend to explore a novel discretetime SMC method for optoelectronic tracking system in this paper.

The remainder of this paper is organized as follows. In Section 2, the discrete-time state space model with regard to one axis of optoelectronic tracking system is constructed mathematically. Section 3 presents the design process of discrete-time GSMC (DGSMC) scheme and the analysis about the performances of control system. Numerical simulation results are shown in Section 4, while the conclusion and the future work are discussed in Section 5.

\section{Problem Description}

Optoelectronic system is a type of servo systems with high precision in position tracking. In this research, each axis of optoelectronic system is driven by a DC torque motor; therefore, it can be mathematically described as [18]

$$
\dot{\mathbf{x}}(t)=\mathbf{A x}(t)+\mathbf{b} u(t),
$$

where,

$$
\mathbf{A}=\left[\begin{array}{cc}
0 & 1 \\
0 & -\frac{B}{J}
\end{array}\right], \quad \mathbf{b}=\left[\begin{array}{c}
0 \\
\frac{1}{J}
\end{array}\right], \quad \mathbf{x}(t)=\left[\begin{array}{c}
\theta(t) \\
\omega(t)
\end{array}\right] .
$$

Moreover, $J$ and $B$ represent the equivalent moment of inertia and the equivalent damping coefficient respectively; $\theta, \omega$, and $u$ represent the angle, the angular speed, and the control input.

Based on the following transformation equations:

$$
\begin{gathered}
\mathbf{F}=e^{\mathbf{A} T_{s}}, \\
\mathbf{g}=\int_{0}^{T_{s}} e^{\mathbf{A} t} \mathbf{b} \mathrm{d} t
\end{gathered}
$$

we can get the discrete-time form of system (1), as shown in

$$
\mathbf{x}(k+1)=\mathbf{F x}(k)+\mathbf{g} u(k),
$$

where

$$
\begin{aligned}
& \mathbf{F}=\left[\begin{array}{cc}
1 & \frac{J}{B}\left(1-e^{-B T_{s} / J}\right) \\
0 & e^{-B T_{s} / J}
\end{array}\right], \\
& \mathbf{g}=\left[\begin{array}{c}
\frac{1}{B}\left[T_{s}+\frac{J}{B}\left(e^{-B T_{s} / J}-1\right)\right] \\
\frac{1}{B}\left(1-e^{-B T_{s} / J}\right)
\end{array}\right] .
\end{aligned}
$$

At this time, the parameter variations and external disturbances are considered in system (4), then (4) is transformed into

$$
\begin{aligned}
\mathbf{x}(k+1)= & {[\mathbf{F}+\Delta \mathbf{F}(k)] \mathbf{x}(k) } \\
& +[\mathbf{g}+\Delta \mathbf{g}(k)] u(k)+\mathbf{d}(k) \\
= & \mathbf{F x}(k)+\mathbf{g} u(k)+\boldsymbol{\delta}(k),
\end{aligned}
$$

where $\Delta \mathbf{F}$ and $\Delta \mathbf{g}$ are the time-varying parameter matrix/vector, $\mathbf{d}$ is the external disturbances vector. Moreover, the comprehensive uncertainties vector is

$$
\boldsymbol{\delta}(k)=\Delta \mathbf{F}(k) \mathbf{x}(k)+\Delta \mathbf{g}(k) u(k)+\mathbf{d}(k) .
$$

The discrete-time model (6) lays the foundation for the next design of DGSMC.

\section{DGSMC Design and Analysis}

The DGSMC is designed for optoelectronic tracking system (6) in this section, and the global reachability and the chattering-free property of control system using DGSMC are also presented.

3.1. Switching Function Design. Define the desired state vector $\mathbf{x}_{d}$ and the state error vector $\mathbf{x}_{e}$, respectively, as follows:

$$
\begin{gathered}
\mathbf{x}_{d}(k)=\left[\begin{array}{ll}
\theta_{d}(k) & \omega_{d}(k)
\end{array}\right]^{T}, \\
\mathbf{x}_{e}(k)=\mathbf{x}_{d}(k)-\mathbf{x}(k),
\end{gathered}
$$

where $\theta_{d}$ and $\omega_{d}$ represent the angle command and the angular speed command, respectively.

We design the nonlinear switching function for system (6) as follows:

$$
s(k)=\mathbf{c x}_{e}(k)-\mathbf{c P}(k) \mathbf{x}_{e}(0),
$$

where

$$
\begin{gathered}
\mathbf{c}=\left[\begin{array}{ll}
c_{1} & 1
\end{array}\right], \quad c_{1}>0 \\
\mathbf{P}(k)=\left[\begin{array}{cc}
p_{1}^{-k} & 0 \\
0 & p_{2}^{-k}
\end{array}\right], \quad\left|p_{1}\right|>1,\left|p_{2}\right|>1 .
\end{gathered}
$$

The vector $\mathbf{c}$ is designed so as to satisfy $\mathbf{c g} \neq 0$. Moreover, at initial time, that is, $k=0$, we can get $s(0)=0$, which means that the state trajectory of control system is set on the sliding surface at initial time, and then the initial reaching time to sliding surface is shorten greatly. 
Under the design of nonlinear switching function (10), the sliding mode motion can be divided into two phases. One phase is that $\mathbf{P}(k)$ converges to $\mathbf{0}$ according to power rule, that is, $\mathbf{c x}_{e}(k)$ converges to 0 , and the duration time of this phase depends on the absolute values of $p_{1}$ and $p_{2}$. The other phase is described by $\mathbf{c x}_{e}(k)=0$, which is similar with the sliding mode motion of SMC method using general linear switching function [19].

3.2. Adaptive Reaching Law Design. The reaching law approach is adopted, and the convenient reaching law is expressed by [20]

$$
\begin{array}{r}
s(k+1)-s(k)=-q T_{s} s(k)-\varepsilon T_{s} \operatorname{sgn}[s(k)], \\
q>0, \varepsilon>0, q T_{s}<1 .
\end{array}
$$

From (12), when system trajectory is far away from sliding surface, the reaching speed mainly depends on the value of $q$. However, when system trajectory is close to sliding surface, the reaching speed mainly depends on the value of $\varepsilon$. Theoretically, larger $q$ and $\varepsilon$ can assure faster reaching speed. Moreover, larger $\varepsilon$ can assure stronger robustness against system uncertainties, but it also brings more intense chattering in control quantity. Therefore, parameter $\varepsilon$ should change adaptively according to the distance from system trajectory to sliding surface.

As a result, we can acquire the adaptive gain of signum term through the following equation:

$$
\varepsilon(k)=\frac{|s(k)|}{\rho}, \quad \rho>0 .
$$

Then, the adaptive discrete-time reaching law is designed as follows:

$$
s(k+1)-s(k)=-q T_{s} s(k)-\frac{|s(k)|}{\rho} T_{s} \operatorname{sgn}[s(k)] .
$$

From [20], when discrete-time reaching law approach is used and the following expression is satisfied:

$$
|s(k)|>\frac{\varepsilon(k) T_{s}}{2-q T_{s}},
$$

the absolute value of $s(k)$ will decrease gradually. Based on (13) and (15), we can get

$$
\varepsilon(k)<\frac{\left(2-q T_{s}\right)|s(k)|}{T_{s}} .
$$

According to (13) and (16), we can deduce that the sample time $T_{s}$ must satisfy

$$
T_{s}<\frac{2 \rho}{1+\rho q} .
$$

Specially, when $\rho=1 / q$ is satisfied, (17) be will transformed into $T_{s}<1 / q$, which is consistent with the condition in (12).
3.3. Controller Design and Analysis. Based on the former nonlinear switching function and adaptive reaching law, the DGSMC will be designed in this subsection.

Assume 1. Sample time $T_{s}$ is small enough to satisfy $T_{s}<$ $\rho /(1+\rho q)$.

Theorem 2. For system (6), choosing switching function (10), the DGSMC law is designed as follows:

$$
\begin{aligned}
u(k)=-(\mathbf{c g})^{-1}\{\mathbf{c}[ & (\mathbf{F}-\mathbf{I}) \mathbf{x}(k)+\Delta \mathbf{P}(k) \mathbf{x}_{e}(0) \\
& \left.-\Delta \mathbf{x}_{d}(k)+\boldsymbol{\delta}(k)\right] \\
& \left.-q T_{s} s(k)-\frac{|s(k)|}{\rho} T_{s} \operatorname{sgn}[s(k)]\right\},
\end{aligned}
$$

where $\mathbf{I}$ is $2 \times 2$ unit matrix, and

$$
\begin{gathered}
\Delta \mathbf{P}(k)=\mathbf{P}(k+1)-\mathbf{P}(k), \\
\Delta \mathbf{x}_{d}(k)=\mathbf{x}_{d}(k+1)-\mathbf{x}_{d}(k) .
\end{gathered}
$$

Then, system trajectory approaches to sliding surface asymptotically, and the chattering at control input is free.

Proof. From (9) and (10), we can get

$$
\begin{aligned}
s(k+1) & =\mathbf{c x}_{e}(k+1)-\mathbf{c P}(k+1) \mathbf{x}_{e}(0) \\
& =-\mathbf{c x}(k+1)+\mathbf{c} \mathbf{x}_{d}(k+1)-\mathbf{c P}(k+1) \mathbf{x}_{e}(0) .
\end{aligned}
$$

Substituting (6) in (20) gives

$$
\begin{aligned}
s(k+1)= & -\mathbf{c F x}(k)-\mathbf{c g} u(k) \\
& -\mathbf{c} \boldsymbol{\delta}(k)+\mathbf{c x}_{d}(k+1)-\mathbf{c P}(k+1) \mathbf{x}_{e}(0) .
\end{aligned}
$$

Utilizing control law (18), (21) can be simplified into

$$
s(k+1)=\left(1-q T_{s}\right) s(k)-\frac{|s(k)|}{\rho} T_{s} \operatorname{sgn}[s(k)] \text {. }
$$

Therefore, we can get the following expression when $s(k) \neq 0$ :

$$
\begin{aligned}
{[s(k} & +1)-s(k)] \operatorname{sgn}[s(k)] \\
& =\left\{-q T_{s} s(k)-\frac{|s(k)|}{\rho} T_{s} \operatorname{sgn}[s(k)]\right\} \operatorname{sgn}[s(k)] \\
& =-\left(q+\frac{1}{\rho}\right) T_{s}|s(k)|<0 .
\end{aligned}
$$

In the premise of Assume 1, the following expression is also acquired:

$$
\begin{aligned}
s(k+1) & s(k) \\
& =\left(1-q T_{s}\right) s^{2}(k)-\frac{|s(k)|}{\rho} T_{s} s(k) \operatorname{sgn}[s(k)] \\
& =\left(1-q T_{s}-\frac{1}{\rho} T_{s}\right) s^{2}(k)>0 .
\end{aligned}
$$


Based on (23)-(24), we can conclude that system trajectory can approach to the sliding surface asymptotically from any initial position, which reflects the global reachability of control system. Moreover, the chattering at control input is avoided effectively.

Note that, in DGSMC law (18), the values of $\mathbf{x}_{d}(k+1)$ and $\boldsymbol{\delta}(k)$ at current time cannot be directly obtained by simple measuring methods, which means that DGSMC law (18) cannot be easily realized in practice. However, we could utilize the known values at former times to estimate the unknown values. That is, we will face the extrapolation problem. Subsequently, the DGSMC law (18) will be improved in the next research.

Assume 3. The changing speeds of system parameters, external disturbances, and tracking signals are much slower than the sampling speed of discrete control system.

In the premise of Assume 3, we can use linear extrapolation method to estimate the values of $\mathbf{x}_{d}(k+1)$ and $\boldsymbol{\delta}(k)$ at current time, respectively.

Consider

$$
\begin{gathered}
\widehat{\mathbf{x}}_{d}(k+1)=2 \mathbf{x}_{d}(k)-\mathbf{x}_{d}(k-1), \\
\widehat{\boldsymbol{\delta}}(k)=2 \boldsymbol{\delta}(k-1)-\boldsymbol{\delta}(k-2) .
\end{gathered}
$$

Therefore, the DGSMC (18) will be transformed into

$$
\begin{aligned}
u(k)=-(\mathbf{c g})^{-1}\{\mathbf{c}[ & (\mathbf{F}-\mathbf{I}) \mathbf{x}(k) \\
& \left.+\Delta \mathbf{P}(k) \mathbf{x}_{e}(0)-\Delta \widehat{\mathbf{x}}_{d}(k)+\widehat{\boldsymbol{\delta}}(k)\right] \\
& \left.-q T_{s} s(k)-\frac{|s(k)|}{\rho} T_{s} \operatorname{sgn}[s(k)]\right\},
\end{aligned}
$$

where

$$
\Delta \widehat{\mathbf{x}}_{d}(k)=\widehat{\mathbf{x}}_{d}(k+1)-\mathbf{x}_{d}(k) .
$$

Obviously, when $T_{s} \rightarrow 0$ exists, there are $\widehat{\mathbf{x}}_{d}(k+1) \rightarrow$ $\mathbf{x}_{d}(k+1)$ and $\widehat{\boldsymbol{\delta}}(k) \rightarrow \boldsymbol{\delta}(k)$. At this time, Theorem 2 is still correct. However, it is impossible to get infinitely-small sample time, which means that the estimation errors must exist. As a result, we need to study the adoption conditions of DGSMC law (26) in next research.

Assume 4. Sample time $T_{s}$ is small enough to satisfy $T_{s}<$ $\rho /(2+\rho q)$.

Based on (7), we can get the following equations:

$$
\begin{gathered}
\boldsymbol{\delta}(k-1)=\mathbf{x}(k)-\mathbf{F} \mathbf{x}(k-1)-\mathbf{g} \boldsymbol{u}(k-1), \\
\boldsymbol{\delta}(k-2)=\mathbf{x}(k-1)-\mathbf{F} \mathbf{x}(k-2)-\mathbf{g} \boldsymbol{u}(k-2) .
\end{gathered}
$$

Therefore, the DGSMC law (26) can be further evolved, and then Theorem 5 is born.
Theorem 5. For system (6), choosing switching function (10), the DGSMC law is designed as follows:

$$
\begin{aligned}
u(k)= & -(\mathbf{c g})^{-1} \\
& \times\left\{\mathbf { c } \left[\sum_{i=0}^{2}\left[\mathbf{U}_{i} \mathbf{x}(k-i)+\mathbf{V}_{i} u(k-i)\right]\right.\right. \\
& \left.+\Delta \mathbf{P}(k) \mathbf{x}_{e}(0)-\Delta \mathbf{x}_{d}(k-1)\right] \\
& \left.-q T_{s} s(k)-\frac{|s(k)|}{\rho} T_{s} \operatorname{sgn}[s(k)]\right\},
\end{aligned}
$$

where

$$
\begin{array}{ccc}
\mathbf{U}_{0}=\mathbf{F}+\mathbf{I}, & \mathbf{U}_{1}=-2 \mathbf{F}-\mathbf{I}, & \mathbf{U}_{2}=\mathbf{F}, \\
\mathbf{V}_{0}=\mathbf{0}, & \mathbf{V}_{1}=-2 \mathbf{g}, & \mathbf{V}_{2}=\mathbf{g} .
\end{array}
$$

If the following condition is satisfied:

$$
\begin{gathered}
0<\rho<\frac{T_{s} s_{\min }}{E_{\max }}, \quad s_{\min }=\min |s(k)|, \\
E_{\text {max }}=\max |E(k)| \neq 0,
\end{gathered}
$$

where the estimation error $E(k)$ is expressed by

$$
E(k)=\mathbf{c}\left[\mathbf{x}_{d}(k+1)-\widehat{\mathbf{x}}_{d}(k+1)\right]+\mathbf{c}[\widehat{\boldsymbol{\delta}}(k)-\boldsymbol{\delta}(k)],
$$

system trajectory will approach to sliding surface asymptotically, and the chattering at control input will be free.

Proof. Similar with Theorem 2, we can firstly get

$$
s(k+1)=\left(1-q T_{s}\right) s(k)-\frac{|s(k)|}{\rho} T_{s} \operatorname{sgn}[s(k)]+E(k) \text {. }
$$

In fact, $E(k)$ is composed by two terms, which is shown as follows:

$$
\begin{gathered}
E_{1}(k)=\mathbf{c}\left[\mathbf{x}_{d}(k+1)-\widehat{\mathbf{x}}_{d}(k+1)\right], \\
E_{2}(k)=\mathbf{c}[\widehat{\boldsymbol{\delta}}(k)-\boldsymbol{\delta}(k)] .
\end{gathered}
$$

The two terms are related with the estimation errors of $\mathbf{x}_{d}(k+$ $1)$ and $\boldsymbol{\delta}(k)$, respectively. 


$$
\begin{aligned}
& \text { Further operations get } \\
& \begin{aligned}
{[s} & (k+1)-s(k)] \operatorname{sgn}[s(k)] \\
= & \left\{-q T_{s} s(k)-\frac{|s(k)|}{\rho} T_{s} \operatorname{sgn}[s(k)]+E(k)\right\} \operatorname{sgn}[s(k)] \\
= & -q T_{s}|s(k)|-\frac{1}{\rho} T_{s}|s(k)|+E(k) \operatorname{sgn}[s(k)], \\
s( & +1) s(k) \\
= & \left(1-q T_{s}-\frac{1}{\rho} T_{s}\right) s^{2}(k)+E(k) s(k) \\
= & \left(1-q T_{s}-\frac{2}{\rho} T_{s}\right) s^{2}(k)+\frac{1}{\rho} T_{s} s^{2}(k)+E(k) s(k) \\
= & \left(1-q T_{s}-\frac{2}{\rho} T_{s}\right) s^{2}(k) \\
& +|s(k)|\left\{\frac{1}{\rho} T_{s}|s(k)|+E(k) \operatorname{sgn}[s(k)]\right\} .
\end{aligned}
\end{aligned}
$$

If condition (31) is satisfied, we can also get

$$
|E(k)|<\frac{T_{s}|s(k)|}{\rho} .
$$

Then, from (35), we can deduce the following expressions:

$$
\begin{gathered}
{[s(k+1)-s(k)] \operatorname{sgn}[s(k)]<0,} \\
s(k+1) s(k)>0 .
\end{gathered}
$$

At this time, we can conclude that system trajectory will approach to sliding surface asymptotically, and the chattering at control input will be free.

From DGSMC law (29), we can use the known information at current time and former times to calculate the control quantity for optoelectronic tracking system; therefore, the proposed DGSMC can be easily implemented in engineering.

\section{Numerical Simulation}

In this section, the simulation results of the proposed DGSMC scheme are compared with those of conventional SMC (CSMC) scheme, with respect to tracking property and chattering degree.

The CSMC still employs the linear extrapolation method to get $\mathbf{x}_{d}(k+1)$, but it introduces the constant gain of signum

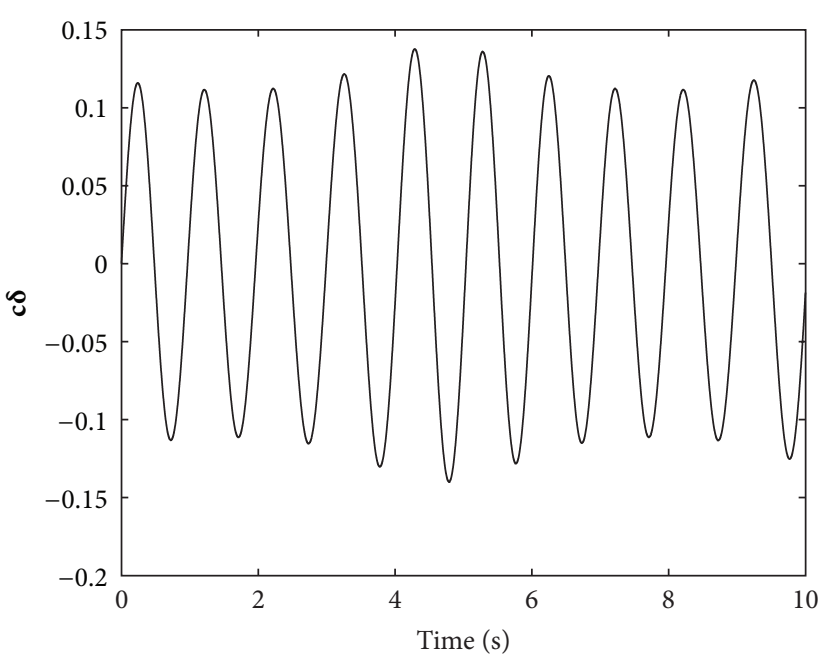

FIGURE 1: The changing curve of uncertainty term.

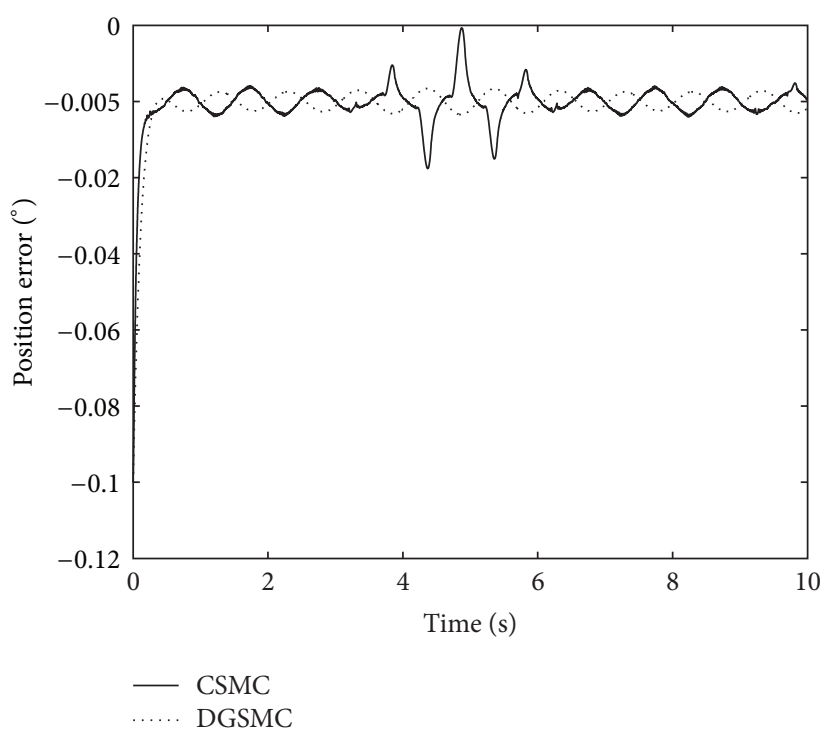

FIGURE 2: The curves of position tracking errors under two control schemes.

term. Assume that the uncertainty term satisfy $|\boldsymbol{c} \delta(k)|<M$, then the CSMC law can be designed as follows:

$$
\begin{aligned}
u(k)= & -(\mathbf{c g})^{-1} \\
& \times\left\{\mathbf{c}\left[\mathbf{x}_{d}(k-1)-2 \mathbf{x}_{d}(k)\right]+\mathbf{c F x}(k)\right. \\
& \left.+\left(1-q T_{s}\right) s(k)-\left(\varepsilon T_{s}+M\right) \operatorname{sgn}[s(k)]\right\}, \\
& s(k)=\mathbf{c x}_{e}(k) .
\end{aligned}
$$

The numerical simulation is carried out on an axis of optoelectronic tracking system, whose parameters are $J=$ $0.0667 \mathrm{~V} /\left(\left(^{\circ}\right) \cdot \mathrm{s}^{-2}\right)$ and $B=0.0833 \mathrm{~V} /\left(\left(^{\circ}\right) \cdot \mathrm{s}^{-1}\right)$. The sample 


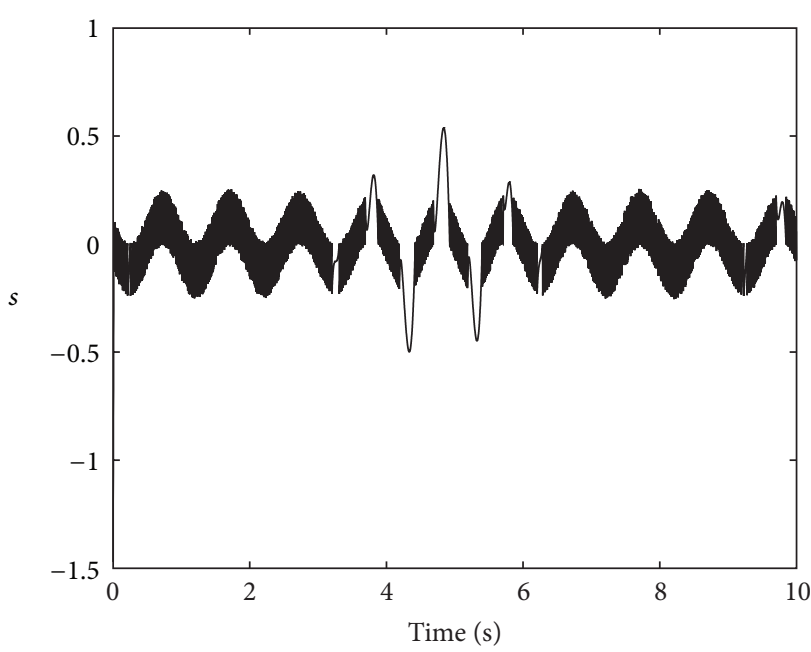

(a) CSMC

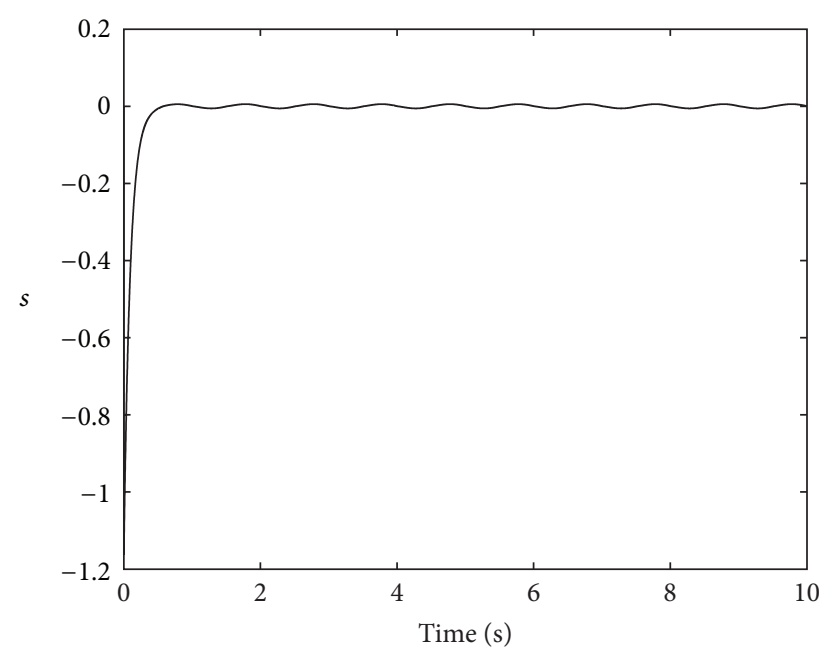

(b) DGSMC

FIGURE 3: The curves of switching functions under two control schemes.

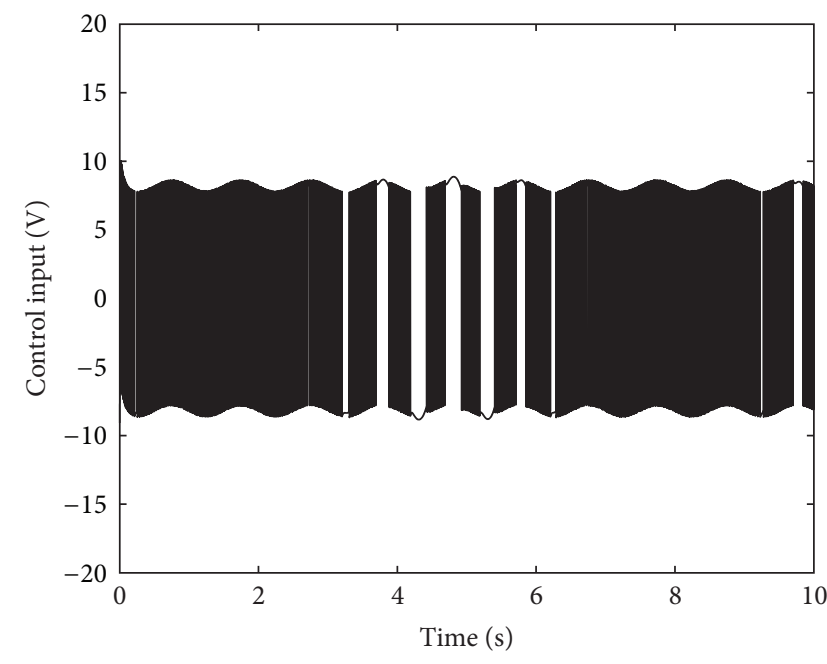

(a) CSMC

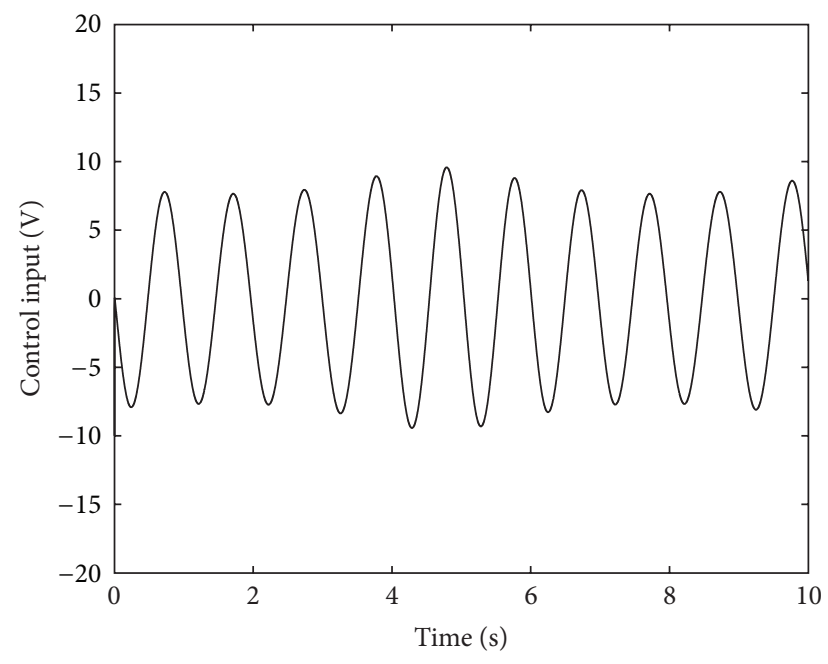

(b) DGSMC

FIgURE 4: The curves of control inputs under two control schemes.

time $T_{s}=0.001 \mathrm{~s}$. The system uncertainties in (6) are given as follows:

$$
\begin{aligned}
& \Delta \mathbf{F}(k)=\left[\begin{array}{cc}
0 & 0 \\
0 & 0.05 \sin \left(k T_{s}\right)
\end{array}\right], \\
& \Delta \mathbf{g}(k)=\left[\begin{array}{c}
0 \\
0.002 \sin \left(k T_{s}\right)
\end{array}\right], \\
& \mathbf{d}(k)=\left[\begin{array}{c}
0 \\
0.12 \sin \left(2 \pi k T_{s}\right)
\end{array}\right] .
\end{aligned}
$$

The coefficients in DGSMC are chosen as

$$
\begin{gathered}
c_{1}=20, \quad p_{1}=10, \quad p_{2}=10, \\
q=10, \quad \rho=2 .
\end{gathered}
$$

The coefficients in CSMC are chosen as

$$
c_{1}=20, \quad q=10, \quad \varepsilon=1, \quad M=0.12 \text {. }
$$

The command signal is chosen as

$$
\theta_{d}(k)=0.1^{\circ} \sin \left(2 \pi k T_{s}\right) .
$$

The initial state vector is $\left[\begin{array}{ll}0.1^{\circ} & 0.1\left(^{\circ}\right) / \mathrm{s}\end{array}\right]^{\mathrm{T}}$. The control voltage is limited between $\pm 10 \mathrm{~V}$ for practical meanings.

The system uncertainties in simulation are directly reflected by uncertainty term $\boldsymbol{c} \boldsymbol{\delta}(k)$, which is illustrated in Figure 1.

Then, the position tracking errors of optoelectronic tracking systems using DGSMC and CSMC are presented in Figure 2.

From Figure 2, we can see that the optoelectronic tracking system using DGSMC possesses smaller position tracking 


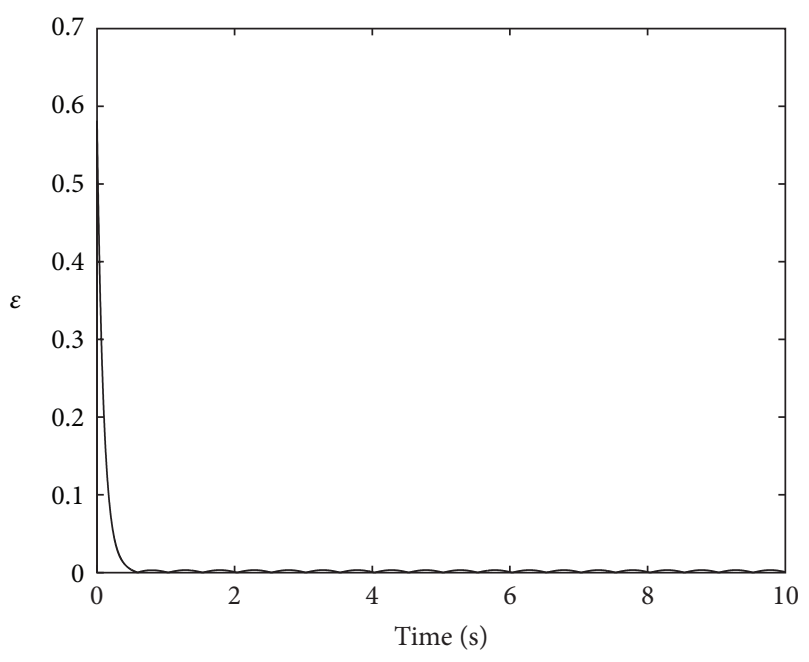

FIGURE 5: The changing curve of adaptive parameter.

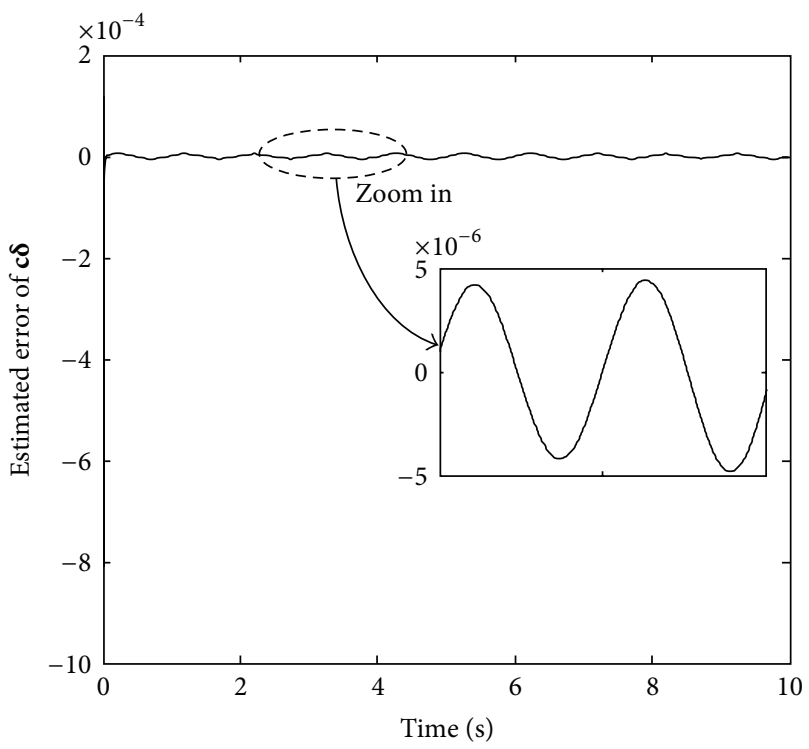

FIGURE 6: The estimated error curve of uncertainty term.

error, compared with CSMC scheme. In Particular, when the big disturbance occurs in control process, the small gain of signum term in CSMC scheme leads to big tracking error. Meanwhile, the tracking error under DGSMC scheme can be maintained at a certain level in despite of the existence of time-varying uncertainties, which directly reflects that the robust property of DGSMC is better than that of CSMC.

In fact, the tracking property of optoelectronic system depends on the convergence property of switching function. Then, the switching functions under two control schemes are illustrated in Figure 3.

From Figure 3, the convergent values of the switching function of DGSMC are much smaller than those of CSMC, and they can keep at a certain level in stable control period. These lead to the better tracking property of DGSMC. Moreover, in switching function of CSMC scheme, there exists high-frequency chattering between positive and negative values, which causes the high-frequency chattering in control input. In addition, the system trajectory under DGSMC is initially set on the designed sliding surface from the simulation data.

In order to analyze the chattering degrees of control inputs under two control schemes, the curves of control inputs are given in Figure 4.

From Figure 4, we can see that the chattering at control input under DGSMC scheme is suppressed more greatly, compared with CSMC scheme. This will bring more protections to optoelectronic tracking plant. Moreover, the energy expenditure and system instability resulting from the highfrequency variations of control voltage can be avoided.

From Figures 2-4, the better comprehensive properties with respect to smaller tracking error, stronger robustness against unknown and time-varying disturbances, and smoother control input are presented, compared with the CSMC scheme.

In fact, the realization of both the small tracking error and the effective suppression against chattering is owed to the adaptive parameter $\varepsilon$ and the estimation mechanism on system uncertainties, which are illustrated in Figures 5 and 6, respectively.

From Figures 3 and 5, the parameter $\varepsilon$ is adapting with the current distance from system trajectory to sliding surface, because the adaptive reaching law (14) is employed. Moreover, from Figure 6, the estimation on system uncertainties is relatively accurate by means of linear extrapolation method, which ensures the effectiveness of DGSMC scheme and enhances the robustness of control system.

Until now, the feasibility and effectiveness of the proposed DGSMC scheme have been verified.

\section{Conclusions}

Based on discrete-time adaptive reaching law and linear extrapolation, the proposed DGSMC scheme can make optoelectronic tracking system robust against parameter perturbations and external disturbances in the whole control period, and then the tracking error of optoelectronic tracking system can be maintained at an expected level in the existence of sustained uncertainties. Moreover, the chattering at control input is not obvious. Therefore, the proposed DGSMC can be used as a new servo control technology applying in optoelectronic tracking system. In addition, the DGSMC algorithm possesses other advantages including simple structure and easy implementation. The experimental verification will be needed in future work. Moreover, when the proposed DGSMC scheme is applied in actual optoelectronic tracking system, how to effectively acquire the difference components of DGSMC law in a noisy environment should be considered and solved. As a result, some more excellent estimation algorithms, such as trackingdifferentiator [21] and approximate differential method [22], can be used as references in further work.

\section{Conflict of Interests}

The authors declare that there is no conflict of interests regarding the publication of this paper. 


\section{Acknowledgments}

This work was supported by the National Basic Research Program (Grant no. 2012CB821200) and the Scientific Research for Colleges and Universities of Inner Mongolia (Grant no. NJZY13143) in China.

\section{References}

[1] V. V. Nikulin, M. Bouzoubaa, V. A. Skormin, and T. E. Busch, "Lyapunov-based decentralized adaptive control for laser beam tracking systems," IEEE Transactions on Aerospace and Electronic Systems, vol. 39, no. 4, pp. 1191-1200, 2003.

[2] P. Orzechowski, N. Chen, S. Gibson, and T.-C. Tsao, "Adaptive control of jitter in a laser beam pointing system," in Proceedings of the American Control Conference, pp. 2700-2705, June 2006.

[3] J. Y. Hung, W. Gao, and J. C. Hung, "Variable structure control: a survey," IEEE Transactions on Industrial Electronics, vol. 40, no. 1, pp. 2-22, 1993.

[4] F.-J. Lin, C.-K. Chang, and P.-K. Huang, "FPGA-based adaptive backstepping sliding-mode control for linear induction motor drive," IEEE Transactions on Power Electronics, vol. 22, no. 4, pp. 1222-1231, 2007.

[5] S.-Y. Chen and F.-J. Lin, "Robust nonsingular terminal slidingmode control for nonlinear magnetic bearing system," IEEE Transactions on Control Systems Technology, vol. 19, no. 3, pp. 636-643, 2011.

[6] J. D. Lee, S. Khoo, and Z. B. Wang, "DSP-based sliding-mode control for electromagnetic-levitation precise-position system," IEEE Transactions on Industrial Informatics, vol. 9, no. 2, pp. 817-827, 2013.

[7] X. H. Mo and Q. X. Lan, "Finite-time integral sliding mode control for motion control of permanent-magnet linear motors," Mathematical Problems in Engineering, vol. 2013, Article ID 567610, 7 pages, 2013.

[8] W. Gao and J. C. Hung, "Variable structure control of nonlinear systems. A new approach," IEEE Transactions on Industrial Electronics, vol. 40, no. 1, pp. 45-55, 1993.

[9] M. I. Bayindir, H. Can, Z. H. Akpolat, M. Ozdemir, and E. Akin, "Application of reaching law approach to the position control of a vector controlled induction motor drive," Electrical Engineering, vol. 87, no. 4, pp. 207-215, 2005.

[10] H.-S. Choi, Y.-H. Park, Y. Cho, and M. Lee, "Global slidingmode control," IEEE Control Systems Magazine, vol. 21, no. 3, pp. 27-35, 2001.

[11] L. Liu, Z. Han, and W. Li, "Global sliding mode control and application in chaotic systems," Nonlinear Dynamics, vol. 56, no. 1-2, pp. 193-198, 2009.

[12] H. Pang and X. Chen, "Global robust optimal sliding mode control for uncertain affine nonlinear systems," Journal of Systems Engineering and Electronics, vol. 20, no. 4, pp. 838-843, 2009.

[13] X. D. Liu, Y. J. Wu, Y. Deng, and S. Xiao, "A global sliding mode controller for missile electromechanical actuator servo system," Proceedings of the Institution of Mechanical Engineers, 2013.

[14] S.-J. Huang and K.-S. Huang, "An adaptive fuzzy sliding-mode controller for sermomechanism disturbance rejection," IEEE Transactions on Industrial Electronics, vol. 48, no. 4, pp. 845852, 2001.

[15] R.-J. Wai, "Fuzzy sliding-mode control using adaptive tuning technique," IEEE Transactions on Industrial Electronics, vol. 54, no. 1, pp. 586-594, 2007.
[16] T. H. Ho and K. K. Ahn, "Speed control of a hydraulic pressure coupling drive using an adaptive fuzzy sliding-mode control," IEEE/ASME Transactions on Mechatronics, vol. 17, no. 5, pp. 976-986, 2011.

[17] X. D. Liu, Y. J. Wu, and B. T. Liu, “The research of adaptive sliding mode controller for motor servo system using fuzzy upper bound on disturbances," International Journal of Control, Automation, and Systems, vol. 10, no. 5, pp. 1064-1069, 2012.

[18] Y. Ren, Z. H. Liu, and R. Zhou, "Application of low speed optoelectronic tracking systems based on sliding mode disturbance observer," Journal of Beijing University of Aeronautics and Astronautics, vol. 39, no. 6, pp. 835-840, 2013.

[19] Y. J. Wu, Y. M. Liu, and W. L. Zhang, "A discrete-time chattering free sliding mode control with multirate sampling method for flight simulator," Mathematical Problems in Engineering, vol. 2013, Article ID 865493, 8 pages, 2013.

[20] W. Gao, Y. Wang, and A. Homaifa, "Discrete-time variable structure control systems," IEEE Transactions on Industrial Electronics, vol. 42, no. 2, pp. 117-122, 1995.

[21] X. Wang, Z. Chen, and G. Yang, "Finite-time-convergent differentiator based on singular perturbation technique," IEEE Transactions on Automatic Control, vol. 52, no. 9, pp. 1731-1737, 2007.

[22] Y. Wu, X. Liu, and D. Tian, "Research of compound controller for flight simulator with disturbance observer," Chinese Journal of Aeronautics, vol. 24, no. 5, pp. 613-621, 2011. 


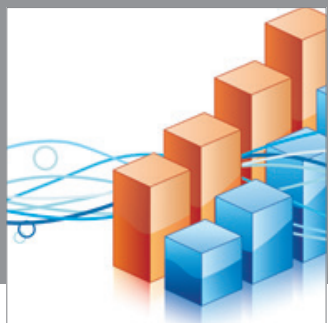

Advances in

Operations Research

mansans

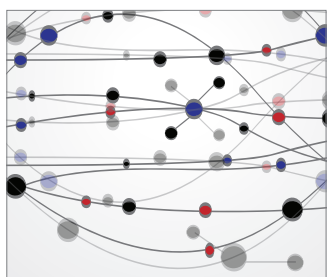

The Scientific World Journal
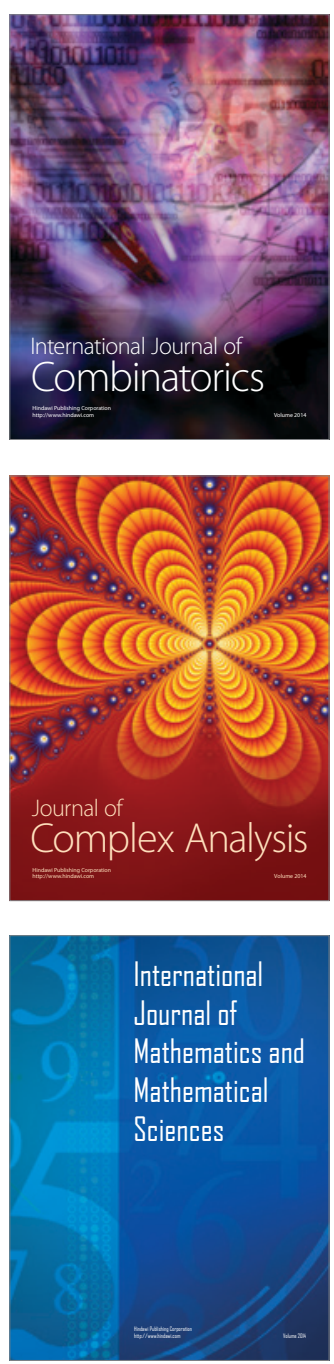
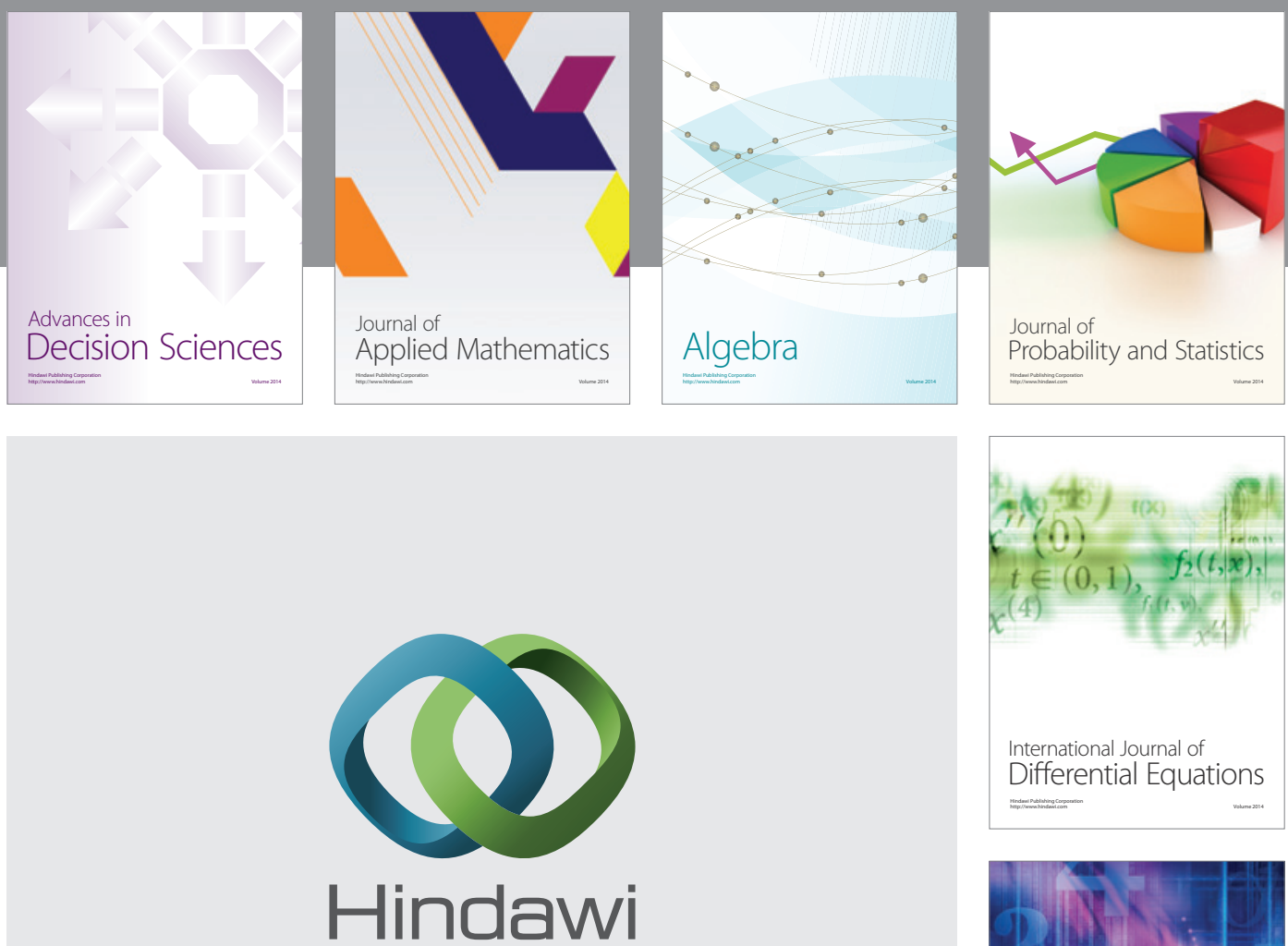

Submit your manuscripts at http://www.hindawi.com
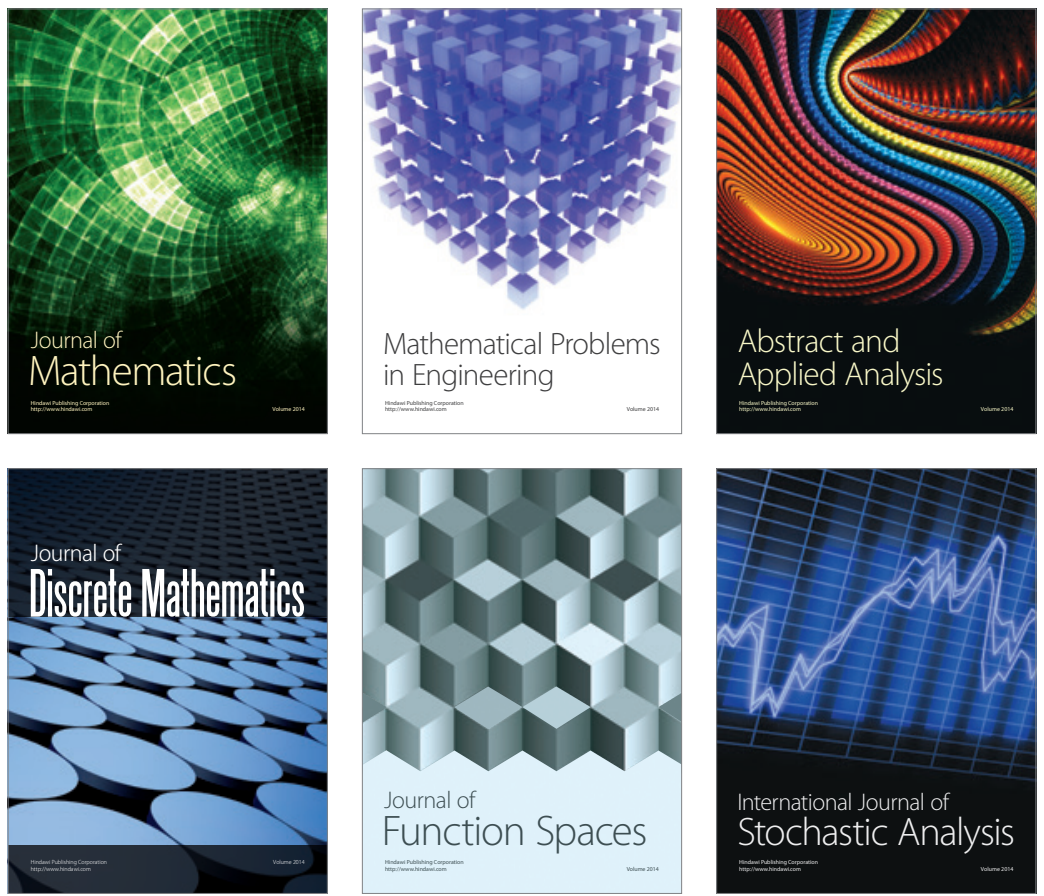

Journal of

Function Spaces

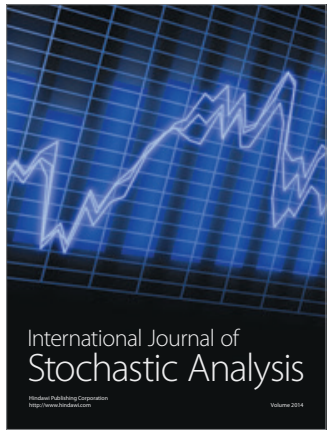

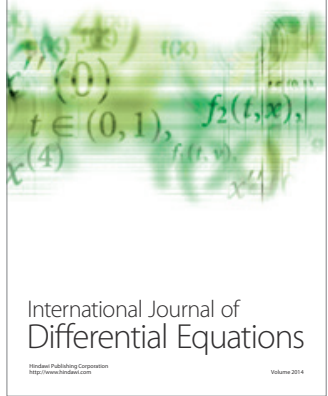
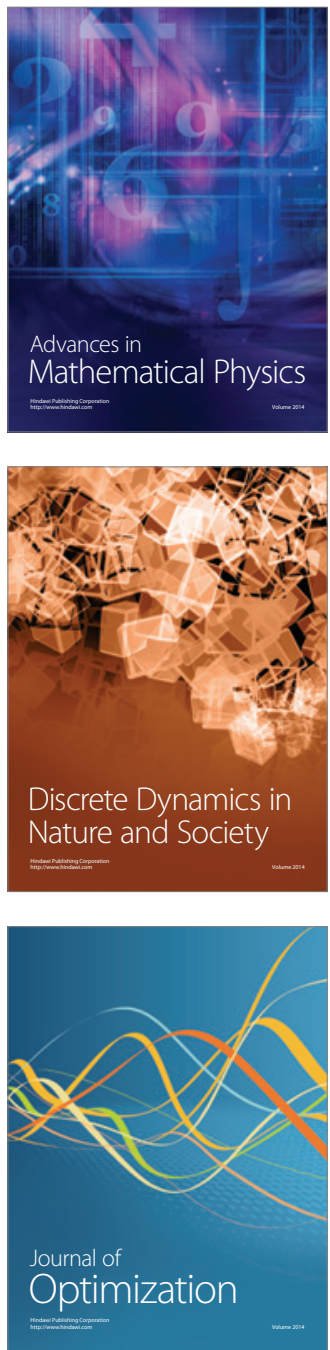